\section{An dydoress}

oN

\section{THE POWERS OF NATURAL RESISTANCE OR THE PERSONAL FACTOR IN DISEASE OF MICROBIC ORIGIN.}

\author{
Being the Annual Oration of the Medical Society of \\ London, delivered on May 26th, 1902,
}

\section{By STEPHEN MACKENZIE, M.D. ABERD., F.R.C.P. LoND., \\ SENIOR PHYSICIAN TO THE LONDON HOSPITAL.}

Mr. Presinent and Gentlamen,- -There can be few amongst us who do not rejoice in the privilege we have of living in an epoch of such progress and mental activity as the present. The great increase of knowledge of the latter half of the last century is found in every branch of science, but it impresses us, perhaps, most in the advance of scientific knowledge in our own profession. It has been brought about by improved means of exact and minute research aided by instruments of increased precision. So that if medicine, in its widest sense, cannot be claimed as an exact science, our methods of dealing with the complex and difficult problems of disease in the living and ever-changing body are as exact as the present state of science permits. Great as have been our advances we cannot doubt that they will be infinitely greater and probably more rapid in the future. Many, however, are dazzled and led away by the apparent completeness of our recently acquired and almost daily increasing knowledge and are inclined to overlook, to neglect, and almost to deride the knowledge which we have acquired by the patient industry and accumulated experience of our predecessors-the work that our fathers have given the best years of their life to accomplish. Our President (Dr, W. H. Allchin) touched upon this point in his address last year. There could be no greater or more inexcusable mistake. Whilst gratefully acknowledging and utilising the newest methods and knowledge as aids to medicine and surgery, theoretical and practical, we must consider them in relation to the observed facts, empirical and scientific, that have been acquired since medicine has been studied as an art or a science. Some of the aphorisms of Hippocrates, the clinical observations of Sydenham, and the pathological observations of John Hunter, are as pregnant of truth and as worthy of being treasured in the memory now as when they were first promulgated, and are in no sense less important than are the most recent discoveries in the laboratories of the bacteriologist and the chemist. It is quite certain that the accurate record of careful observations of the phenomena of disease will always be valuable additions to medical knowledge, whether or not capable of explanation or use at the time they were made. We see striking illustrations of this in the writings of the three great Guy's physicians-Bright, Addison, and Hodgkin. There have been many stages in arriving at the knowledge which we at present possess. The progress of morbid anatomy and the revelations of the bacteriologist and chemist have given great precision to our knowledge and have opened out new methods of research and treatment. The task which I have set myself on the present occasion is to point out that whilst availing ourselves of al scientific discoveries we must not overlook or forget the valuable knowledge which we already possess and may still further acquire by careful, patient, and persevering clinical work. The tendency of the medical mind at the present time seems to be to attach too great importance to the germ or seed and too little to that of the body or soil in diseases of microbic origin. I propose to pass under review a few diseases of this class and to endeavour to point out the share or relative importance of these two factors in such diseases.

\section{INFLUENZA.}

Let us first take the case of influenza. It presents the extreme end of a series of infectious diseases-i.e., diseases of microbic origin. It prevails pandemically at irregular and often long intervals, usually followed by epidemics for some No 4109 . years. When pandemic it is remarkable for the number of persons who are attacked and the rapidity with which it spreads from country to country. Neither race nor class, sex nor age, afford exemption. It is so widely spread when pandemic that very few escape, though probably a very few are immune, and these attribute their escape to some nostrum or the disinfectant most in fashion. At these times the seed is every where and almost everywhere it finds a congenial soil, so that the natural resistance or personal factor, as far as susceptibility goes, is practically nil. Not so the effect; though said to be the smallest bacillus known, like the Biblical grain of mustard-seed it gives rise to very great results. In the aged, in those suffering from pulmonary and cardiac disease, and in those devitalised from any cause, it is a very serious malady often leading to a fatal issue. Though in the robust it is often apparently a comparatively trivial disease this generation has learned that it is to be treated with great respect and that apparently slight cases neglected may lead to very grave results. We have learnt, too, that it may be attended with very serious and dangerous complications, such as abscess of the brain, purulent otitis, phlebitis, affections of the heart and nervous system, \&c. Whether these complications are the direct effect of Pfeiffer's bacillus or are due to a mixed infection has not been fully worked out.

The subject of mixed infections raises a very interesting question of great importance in several of the infectious diseases, indicating that the seed is not always pure or that the tissues the resistance of which has been broken down by one microbe fall a ready prey to others, but into this point I do not propose to enter.

Lastly, we have learnt that the effects of an attack may be of prolonged duration, continuing even for years. Whether they depend on tissue changes effected by the direct action of the bacillus or its toxins in the attack or the latter surviving long after the attack of influenza is uncertain, but we see in diphtheria the supervention of paralysis some weeks after the acute stage has passed off, and in syphilis the occurrence of nervous lesions years after all other active manifestations which we recognise as directly syphilitic have ceased to present themselves. Let us turn now to another subjecttuberculosis.

\section{TUBerculosis}

When I began the study of medicine we were still in the throes of battle as to what was tubercle and what tubercle was. The unitarian doctrine of Laennec did not by any means meet with universal assent. The microscope was pressed into service to decide what was the essential constitution of tubercle and diligent search was made for the "tubercle cell" as there had been for the "cancer cell," as fruitless in the one case as in the other. Then followed the epoch in which Villemin showed that tubercle was inoculable, though many pitfalls were encountered in establishing this fact. Finally, in 1882, Koch was able to demonstrate that the specific cause of tuberculosis in all its various forms was a micro-organism, the bacillus tuberculosis, that it was present in every tuberculous lesion, and that without this there was not any tuberculosis. The gropings to establish its nature, often highly scientific, sometimes blind, were at an end ; the efforts of many of the keenest and ablest workers to understand its morbid anatomy and pathology were harmonised and their errors were corrected and we obtained an absolute criterion to which we could appeal to decide what was tuberculous. It was left to the clinician and sanitarian to apply this knowledge in the treatment and prevention of disease. With the aid of the bacteriologist we have learned a great deal of the life of the tubercle bacillus both inside and outside the body and of the ways by which tuberculous persons become affected. But in this respect the tendency has been perhaps to concentrate attention on the microbe and to devote less consideration than its importance demands to the human host in which it finds a lodgment. This is seen not so much in the deadhouse where the gross changes in the organs are forced on our notice as in the wider aspects of the subject which deal with the causation and prevention of tuberculosis. We are apt to think too much of the seed and too little of the soil. We have learned since Koch's discovery of the bacillus that from many sufferers from tuberculosis, more especially in pulmonary phthisis, the secretions or discharges given forth contain the bacillus in an active condition and that the clothes and bandkerchiefs and the beds and the floors and walls of the dwellings of the sick are contaminated in this manner so as to be a source of danger to 
others. These secretions and discharges undergo desiccation and are commingled with other particles constituting dust which are inhaled with the air we respire, and haply finding in the respiratory or digestive organs conditions favourable for their growth increase and multiply and bring about tuberculosis. We have reasons for believing that casual bacilli may not only reach the respiratory and digestive organs but may make their way by the bloodvessels into practically all parts of the body where, in favouring conditions, they set up tuberculous processes. Thus are accounted for cases of tuberculous meningitis, tubercle of the kidney, \&c. We have learned that the bacillus tuberculosis may be contained in the milk, the butter, the cheese, and the flesh which we consume as food. In recent years great efforts have been made to exclude this mode of infection by the extermination of tuberculosis in cattle and the destruction of food-stuffs derived from infected bovines. Like a bolt from the blue came the recent pronouncement of Koch himself at the late British Congress on Tuberculosis that the risk of transference of tuberculosis from cattle to human beings was so slight as to be a negligible quantity. The gauntlet thus thrown down was immediately taken up. A Royal Commission has been appointed to consider and to report on the subject. Already evidence has been tendered that such transference does take place, a fact not disputed by Koch himself. Many must admit that the evidence not only as to the fact but also of the frequency with which tuberculosis is derived from bovines is in need of revision and consideration. Dieudonné, Cattle, Shennan, Still, Carr, and Colman have shown that in the tuberculosis of infants the primary seat of infection in most cases is through the respiratory and not the digestive tract, and further Koch in his address to the Congress said: "It is just as likely that these cases of intestinal tuberculosis were caused by the widely propagated bacilli of human tuberculosis." Cattle writes : "It is not only milk that goes into the mouths of children ; they grub about on all-fours, plastering their fingers with the tuberculous dust hidden away in inaccessible corners and then put their fingers in their mouths." It is more than possible that the danger of infection from food has been over-estimated. It is none the less our duty to use all the measures which experience dictates to prevent the entrance of the bacilli by this route, and we should be guilty of folly if we relaxed the measures of prevention now in use until we have a decisive verdict on this point.

But it is of the soil in which the tuberculous seed grows that we have also to take account. We have learned that the bacillus tuberculosis is so widely disseminated that no one is exempt from the risk of infection. The dust inhaled in many dwellings, in public rooms, in vehicles, and in the streets is often contaminated with the bacillus. Strauss has shown that in cotton-wool plugs in the nostrils of those in attendance at the Charité Hospital, Paris, tubercle bacilli were found in nearly a third of the cases examined and that they proved infective to animals. Dieudonné found tubercle bacilli and pyogenic cocci on the hands and in the noses of children from the age of nine months to two and a half years whose fathers or mothers were the subjects of tuberculosis. Though the protective arrangements at the entrance to the respiratory passages to some extent safeguards the entrance of the bacilli, this protection is by no means complete, and when adenoids or some malformation of the nostrils compel oral breathing it is very imperfect. This is shown by the great frequency of pulmonary tuberculosis. Post-mortem experiences reveal to us that tuberculous changes are found in the lungs and other parts of persons dying from various nontuberculous diseases or killed by accident-placed by different writers at from 30 to 90 per cent. - but which have become arrested and obsolete. So that not only are all liable to become infected, but we know that in a much larger number of persons than was formerly imagined actual infection does take place. But we learn from this fact also that the infection may be but slight and temporary, and that within the body and in the tissues there exist physiological mechanisms that arrest the invasion and bring it to a standstill. We know also that where the risk of infection must be considerable, as in the aggregations of consumptives in hospitals, very few who move and live among them contract the disease. Though the experience of all hospitals has not been so favourable as has that of the Royal Hospital for Consumption at Brompton it has been stated that over a considerable number of years the incidence of phthisis amongst the physicians, nurses, and servants of that instituion has not been greater than that of the general population

Thus it is demonstrated that the human soil is not in all persons a favourable one for the growth of the tuberculous seed, and that the human organism has a considerable power of resistance and self-protection, very happily illustrated by Osler by the "Parable of the Sower." Dr. W. Howship Dickinson has within the last month published an able and interesting sermon with St. Matthew's Parable of the Sower for his text. Dr. Dickinson has treated the subject of tuberculosis on almost exactly the same lines as myself but in his own peculiarly felicitous manner and literary style. It is equally obvious from the great frequency of tuberculous diseases that all are not equally fortunate in this respecti.e., in the powers of natural resistance; or, in other words, some are more vulnerable than others. Mankind is naturally resistant to tuberculosis (Kanthack). On what this vulnerability immediately depends we need not here discuss, but we know a great deal of the circumstances in which tuberculosis occurs. We know that it may occur at any age, that its various forms have special age proclivities, and that the majority of deaths from tuberculcus affections, as a whole, occur between 20 and 30 years of age. We know by the writings of our predecessors and by our own experience that many inherit and some acquire a tendency to tuberculosis. There can be no doubt that the progeny of the tuberculous are much more liable to tuberculosis than are those in whose family no such strain runs. On what that predisposition depends opinions may differ and we have no absolute knowledge. Whilst congenital tuberculosis, whether placental or germinative, is an established fact, it is extremely rare, but most who are born of tuberculous parents and become tuberculous are not, we believe, actually so at birth. Inheritance in bovine tuberculosis is believed to be answerable for more than one-half of its very considerable mortality. We are often able to detect the tendency by their conformation and we know that they often show a marked tendency to catarrh. I do not discuss the nature and significance of what used to be called the strumous or scrofulous diathesis. We are also quite aware that pulmonary and other forms of tuberculosis do occur in those of robust physique with well-formed chests, but this does not detract from the importance of our experience that the delicate and the frail or those of feeble vitality are the most prone to become tuberculous. The great liability to catarrh in the delicate offspring of tuberculous parents by causing inflammation of the air-passages doubtless weakens their powers of resistance to the tuberculous bacillus which is nearly always at hand. Alcohol again is a predisponent. How often do we hear in taking the history of a family, otherwise cleari as regards tuberculosis, the statement that a relative died from consumption but that "he gave way to drink"-an explanation that in the minds of his family this was the cause of his becoming tuberculous? It has been shown that those who are intemperate or who have opportunities of drinking show a higher mortality from phthisis than do others and that the children of the intemperate are more prone to become tuberculous than are those of the temperate. No doubt through alcohol persons acquire or inherit a lessened power of resistance to tuberculosis. Diseases like measles and whooping-cough, which are often complicated with oroncho-pneumonia, and diabetes, which greatly reduces the powers of vital resistance, may open the door to tuberculosis.

In the exactness of our recently-acquired knowledge of the pathology there has been a tendency, as I have already stated, to deal lightly with the accumulated experience of the past and to set little esteem upon the laborious work of the great masters of medicine. A timely protest against this tendency has been recently made by Sir Dyce Duckworth in his admirable address on "The Personal Factor in Tuberculosis" which deals with this important subject with an ability and length that I cannot command and which $I$ have read since writing this oration. The personal factor in the clinical aspect and in the pathology of tuberculosis is of quite equal importance to that of the microbe essential as that is for the production of tubercle. "The microbe," writes Professor Vaugeley of Bordeaux, "is nothing whilst the organism in which it thrives is the main factor." How thoroughly this is borne out in the treatment of tuberculous diseases. When once the seed has taken a firm root in the body we cannot with certainty at the present time kill it. It may grow or it may wither, we may cut it out when it is purely local and accessible, in some parts we may destroy it by 
Finsen's light treatment or Roentgen rays when it is confined to the skin and superficial mucous membranes; but when deeply seated we at present know of no remedy which will certainly destroy the virus, not even lachnanthes. I say at present because I do not think we ought to be hopeless of effective results from tuberculins or antitoxic serums. It is true that the hopes that were raised by Koch's first tuberculin, prematurely published and boomed by the lay press, did not realise the expectations that were excited. No doubt the pendulum swung round too completely and the first trials insufficiently tested or used only in the manner then advised did not offer encouraging results. Nor has general experience, so far as it has been employed, been much more encouraging as regards Tuberculin $R$. Koch has introduced a still newer preparation and there are some who hope that with modifications and in smaller doses distributed over a considerable period, especially in early cases of phthisis, it may yet prove effective. Petruschky claims to have obtained good results by much smaller doses than were originally employed and extending over several courses with intermissions The method is scientific and is supported by oux experience in rabies, tetanus, and diphtheria. Some remedies, such as creasote and guaiacol, of undoubted service in the treatment of phthisis, though not strictly bactericidal in doses that can be safely administered, may possibly diminish the activity of the bacilli and lessen or prevent secondary tuberculosis of the digestive tract by their action on the bacilli in swallowed sputa, but in the opinion of many they only do good by their action on mucous membranes and by lessening cough and expectoration. We disinfect or destroy the sputa and other discharges from the tuberculous, not for the good of the patient, but to prevent the dissemination of the disease to others. Practically our treatment of tuberculous diseases that are not accessible to the surgeon consists in increasing the vigour and resistance of the patient, by life in the open air, abundance of sunlight which is of great importance as its chemical rays are strongly bactericidal, thorough ventilation of the apartments of the sick, and by choosing a climate especially suitable for each case when practicable, abundant, very nourishing and oleaginous food, and by strict attention to general and personal hygiene. In fact, our principal object and power, whether we recognise it or not, is to endeavour by every means in our hands to raise the natural resistance of the body to the microbe-to devote our attention to rendering the soil an unfavourable one to the growth of the tubercle bacillus. It is encouraging and sometimes astonishing how successful our efforts are in this direction, especially when the circumstances of the patient allow of the full use of the means experience has taught us. It is, however, I think, too great a stretch of the truth to say as has been said that "tuberculosis is one of the most curable of diseases." The wise do not often talk of "curing" tuberculosis. Tubercalosis may become arrested under our treatment, and often without it, but we can never be sure that a quiescent or obsolescent tuberculous process may not resume activity, like Mont Pelée, with eruptive violence under certain circumstances. We welcome and admire the princely gifts of Sir Ernest Cassell and other philanthropists for the establishment of sanatoriums for the treatment of the consumptive, and we recognise the duty of municipal and other bodies taking steps for carrying out the "open-air treatment" of phthisis so as to extend to the less affluent the benefits which otherwise are confined to the rich or well-to-do. Though the open-air treatment was first practised in England by Dr. Boddington it did not meet with general adoption and this country is at the present time behind others in the means for carrying it out. There is now, however, an awakening to the importance of this method of treatment.

A point, however, to which I most particularly desire to draw attention is that the undoubted and great diminution in the mortality from consumption that has been going on during the last 50 years, not only in this country, but also in other countries where statistical evidence is available preceded the almost exact knowledge which we have now obtained of the pathology of tuberculosis and the discovery of the bacillus. The great value of the work of the late Sir George Buchanan should always be remembered in connexion with his reports on the conditions under which consumption was most prevalent. This striking diminution in the mortality of consumption has been steadily going on under many unfavourable conditions, before any effective measures were taken for disinfecting or destroying the sputa or separating the sick from the healthy. During the greater part of this time in numerous cases husband and wife, mother and daughter, sister and sister were occupying the same bed when one has been the subject of consumption. It has gone on, too, in spite of the great migration from the country to the towns. There can be no doubt that this lessening of consumption has been due to improved sanitation, to better draining of the land, to improved disposal of sewage, and to the better housing, clothing, and feeding of the general population. Of course, we recognise the great influence of modern surgery which, dealing with tuberculosis of the bones and the joints, the glands and the serous cavities, has saved a great number of tuberculous subjects from becoming consumptive. The greatest triumphs in tuberculosis as in other diseases have been in prevention.

What lessons do we learn from this general survey of the share taken by the seed and the soil of tuberculosis? First, that we have to prevent or lessen the diffusion of the disease by preventing the dissemination of the tuberculous germs. This is to be done by segregation of the sick either in institutions especially arranged for their care, or when this is impossible by providing that they live in their homes in well-ventilated apartments not occupied by the healthy. We have also to secure the destruction or disinfection of the sputa and other discharges containing the virus and the subsequent disinfection of the rooms that tuberculous subjects have occupied. These measures we are all keenly alive to and we have to continue and to increase our efforts to this end. But we have in addition to taking all means in our power to prevent the propagation and dissemination of the seed not to lose sight of the soil in which it breeds. This is the second direction in which efforts must be made if we are to succeed to lessen still further the prevalence and mortality of tuberculous diseases. What we have to do in this respect is obvious but not easy. We have to increase the natural powers of resistance to the action of the specific microbe both in the individual and in the general population. We have to prevent overcrowding and to improve the dwellings of the working-classes and the poor. Poverty is a powerful predisponent to disease of most kinds but especially to tuberculosis. Koch says: "The breeding-places of the tubercle bacillus are in the slums." Sir J. Crichton Browne has said: "Tubercuiosis stands in inverse ratio to the rateable value of the property." I remember many years ago before the discovery of the bacillus someone pressing the late Dr. H. G. Sutton to define what tubercle really was. He replied : "Tubercle is making shirts at 5s. a week." We cannot make the poor rich but it is our duty no less as citizens than as physicians to see that the poor and the working-classes are decently housed and that their dwellings are kept in a proper sanitary state. We must bring the pressure of public opinion to bear upon municipalities, urban and rural councils, to improve the conditions under which the poor live. Rich and poor alike must be still further educated as to the imperative necessity of fresh air by means of the "open window" or more scientific methods of ventilation. We must secure not only for the dwelling but for all places where people congregate-workshops, factories, churches, and theatres-that they do not respire re-breathed and contaminated air. We must use our influence as medical men to prevent consumptives from marrying and propagating offspring predisposed to the disease; we must continue to urge temperance; we must endeavour to rear a population of more robust physique, offering a more resistant soil to the seeds of tuberculosis. What we have to aim at is the improvement of the race. This is a very high, and to some it may seem a transcendental, ideal, involving great economic and social problems. But the material prosperity of the country has steadily increased. The poor are better housed, clothed, and fed than they were formerly and we bave the encouragement of the past in the last half century teaching that tuberculous diseases have materially diminished by the efforts that have been made in these directions.

\section{Rhelmatic Fever.}

I pass on to the consideration of rheumatic fever. Here our ground is less secure, as the evidence of its bacterial origin is not absolutely admitted at the present moment, though it appears to be within almost immediate realisation. Rheumatic fever is a common disease in this country. It has received a great deal of attention at the hands of medical observers and has exercised the minds of most 
of the thinkers in our profession. The state of our knowledge 30 or $40^{\circ}$ years ago may, I think, be fairly summarised by saying that it was believed and taught that rheumatic fever was an acute non-purulent inflammation of many joints and of fibrous tissues, attended by fever and acid sweats, and liable to be complicated by endocarditis and pericarditis; that it was due to an acid in the blood and was to be treated by alkalies. A nervous theory was put forward to explain some of the symptoms but it never took firm root. Prout suggested that lactic acid was the cause of the disease and $\mathrm{B}$. W. Richardson endeavoured to prove this experimentally, but this evidence is not accepted. Latham a few years ago explained how lactic acid might be formed in the body. The acid theory was at one time so firmly established as to appear impregnable. It was, however, but a remnant of the humoral pathology that at one time dominated medicine. The faith of most was shaken when Gull and Sutton published their well-known paper on the Treatment of Rheumatic Fever by Mint-water. They revealed to us almost for the first time the natural history of the disease untreated by drugs and showed that it ran very much the same course and lasted about the same time as when treated by alkalies or other active medicines. This was of course before the introduction of salicin and salicyl compounds into the treatment of rheumatic fever. The kindly banter of Wilks who said that "the use of alkalies in rheumatic fever was to remove the idea of acidity from the mind of the doctor" probably did not a little to help in the same direction. We have since learned by the gradual growth of medical knowledge to take a wider view of the nature of rheumatism. The original conceptions were founded on descriptions of the disease as seen in the adult, but the study of the disease in childhood has enlarged our grasp of its clinical features, and we know that whilst acidity of the urine and the sweat is characteristic of rheumatic fever it occurs also in other acute febrile diseases. But even if an acidity of blood or rather a diminished alkalinity of the blood is true it has still to be ascertained how this is brought about. It is interesting to note that Poynton and Paine found that the organism which they have found in rheumatic fever grows best in an acid medium. We have learned that rheumatism, like tuberculosis, is often an inherited or family disease, that it has many expressions besides the arthritis, and that many conditions such as endo- and pericarditis, some cases of pleurisy, several affections of the skin, tonsillitis, and chorea may occur independently of arthritis and have as much right to be regarded as rheumatic as the joint affection. I am aware that this position is not universally admitted, but though the difficulties of obtaining evidence on this point are great it is those who have devoted most attention to the subject who, as a rule, are most convinced of its truth. But those who hold this view, equally with those who do not see the facts in the same light, have always recognised that there must be another factor besides predisposition to account for the occurrence of rheumatism. What this tertium quid is has exercised considerable thought and speculation. The influence of the prevailing pathological doctrine has naturally suggested the possibility of a microbe. This is no new view but was maintained years ago by Hueber, Strümpell, and others. It is admitted or suggested that many or most of the clinical phenomena connected with this disease fit in with the microbic theory and that the morbid anatomy is not opposed to it. Rheumatism is mainly a disease of the first half of life ; age exercises a great influence on the form in which it manifests itself. The acute febrile and self-limited course of rheumatic fever corresponds to that observed in other fevers of ascertained microbic origin, whilst the non-febrile or only slightly febrile course of some of the conditions claimed to be rheumatic, such as chorea, is paralleled by diphtheritic paralysis and other nervous affections due to toxins. The occurrence of post scarlet fever rheumatism appears to be an example of "the door being opened " (to use the happy expression of Sir William Broadbent) by the former to the latter, as it is by influenza to typhoid fever. The occurrence of several cases of rheumatism in its various forms in succession in the same house, not confined to members of the same family but in subsequent occupiers, recorded by Mantle and other observers, is strongly in favour of the infective - that is, microbicnature of rheumatism. Newsholme's valuable work points in the same direction. He has pointed out the epidemic or semi-epidemic prevalence of rheumatism. He found that this is associated with lowness of subsoil water and relatively high temperature of the earth, conditions which either probably influence microbic growth or dissemination. Seasonal influence is considerable, as in typhoid fever and cholera, the greater number of cases of rheumatic fever in London occurring in October and November. Rheumatic fever, sike typhoid, confers no immunity to subsequent attacks. On the contrary, a person who has once had rheumatism in any of its forms is extremely likely to have it again, but whether this is due to increased proclivity or simply indicates personal predisposition there is no evidence to show. Several observers, such as Mantle, Achelme, and others, have found micro-organisms in the inflamed joints and blood in rheumatic fever, but their results were not pushed to the point of absolute certainty. Wassermann, Westphal, and Malkoff abroad, and Poynton and Paine in this country, have found a diplococcus which agrees in its general characters. The two llatter have obtained this diplococcus in pure culture from human rheumatic lesions and have reproduced by intravenous inoculation in the rabbit endocarditis, pericarditis, carditis, pleurisy, pneumonia, nodules, and a nervous condition resembling chorea, as well as polyarthritis and inflammation of the sheaths of the tendons, and found the same organisms in the induced lesions.

An observation of great interest made by Poynton and Paine is that in a culture from a "sore-throat" in a patient with muscular pains, a history of a previous attack of rheumatic fever, and valvular disease the diplococcus was found. It was isolated and cultivated and was injected intravenously into a rabbit, producing in that animal mitral valvulitis and great dilatation of the heart. This observation and the fact that sore-throat so commonly occurs as an early symptom of rheumatic fever suggest that the usual point of entrance of the virus is the tonsil or the pharynx. The observations of Poynton and Paine, especially the experimental evidence, appear so complete as to be convincing. We require further confirmation of these observations before the microbic theory can be said to be established beyond dispute, but Osler and Allchin have boldly transferred rheumatic fever to the category of acute infectious diseases. If confirmed, as I believe these researches will be, what a valuable aid they will be to the precise understanding of the pathology of rheumatism. When the seed is fully identified we may hope to learn from its life-history outside the human body means for the prevention and treatment of this disease.

But I wish to point out that if the bacterial nature of rheumatic fever is fully established the patient work on the clinical side will not have been thrown away, that we shall still have to take account of the soil no less than of the seed, and, as in tuberculosis, the personal factor will remain as important in the future as it has been in the past. If the bacterial nature of rheumatism is conclusively proved we shall still have to take into account individual liability to the disease and to recognise that whilst some persons exposed to wet and cold, especially when combined with fatigue and exhaustion, escape, others similarly exposed are prcne to contract rheumatism. Of course, it is well known that many cases occur without such exposure. But Pasteur, Wagner, and others have shown that by exposure to cold fowls (naturally immune) become susceptible to anthrax in fection, and Monti has demonstrated that dogs, naturally more resistant than man to the pneumococcus, are taken il and die from pneumococcic infection solely when they are inoculated after giving them a very cold bath. These facts are suggestive in connexion with the microbic nature of rheumatism. We shall continue to find that the predisposition runs in families and is usually inherited. We shall, in fact, find that the soil is no less essential for the production of rheumatism than is the seed.

\section{Appendicitis, or Perityphlitis.}

Appendicitis, or perityphlitis, offers an interesting and instructive study, clinically and pathologically, in connexion with the theme which we are considering. From being regarded, as it was when I commenced the study of medicine, as a rather rare condition it has come to be regarded as a very common one not only by the profession but by the public. Whilst the less acute or less severe forms have been recognised for a long time we have in recent years learnt that the very acute and fulminating cases due to ulceration and sloughing of the appendix causing abscess and perforation account for a great many obscure cases of peritonitis, the nature of which was unsuspected and misunderstood. The increase of our knowledge on the subject has 
reached a high degree of precision through the observations of the surgeon, the pathologist, and the bacteriologist. It was, I believe, Sir Samuel Wilks who, in the first edition of his work on Morbid Anatomy and Pathology, emphasised the fact that in inflammation and abscess in the right iliac regionperityphlitis-it was usually the vermiform appendix that was at fault and not the cæcum as was then generally taught. To the surgeons in this country and in America we owe the great extension of our knowledge that in inflammatory affections of the abdomen the appendix was at fault with a frequency not previously imagined and they have pointed out the anatomical conditions in the appendix that favour and predispose the appendix to disease. The bacteriologists have determined the micro-organisms that play such an important rôle as the excitors of the inflammation and its consequences in the appendix. Thus, on the one hand, we have learned that micro-organisms are always concerned in the inflammation, ulceration, sloughing, and necrosis that occur in appendicitis. The bacillus coli communis appears to be the most common-a bacterium in ordinary circumstances saprophytic and harmless but which may become virulent and deadly when growing in diseased tissues. More rarely the pus cocci, the streptococcus pyogenes, the staphylococcus, the proteus, and bacillus pyocyaneus are concerned, and the bacillus typhosus has been found to be the organism at fault. On the other hand, we have learnt that peculiarities of position and anatomical formation of the appendix and its mesentery tend to produce contortions and contractions and interference with the circulation in the appendix which prevent the discharge of its secretions, favour the formation of fæcal concretions, and prevent the expulsion of foreign bodies accidentally entering its canal. In one of these ways the vitality of this portion of the intestinal tract, probably owing to its being a vestigial remnant never so high as other parts of the intestine with active functions to perform, becomes impaired. The organisms in the appendix find a medium which enables them to grow and to multiply, often with intense virulence, by which they inflame or destroy the appendix and pass through its walls to the peritoneum and other contiguous parts. Sir Frederick Treves tells us that "it is impossible not to be struck with the fact that perityphlitis runs often in families," not so much as an inherited disease as a family disease. It is much more frequent in the second and third decennia and in the male sex.

So we find the seed and the soil in appendicitis the excitors of active disease and the predisposing conditions-the personal factor in this disease-each essential for its occurrence. But whereas in tuberculosis and rheumatism we are often able to detect the mischief by the history, the patient's appearance, the evidence of individual predisposition, there is nothing in appendicitis that reveals a tendency to this condition until the occurrence of an attack of appendicitis. Moreover, whilst the tendency to most of the infective diseases is a general one more or less distributed throughout the system, though in each there is a favourite mode of entrance of the virus, here it is an entirely local one extending over an area of only a few inches.

Reviewing the group of diseases which I have selected to illustrate my thesis we have seen that in each there are two factors-the soil and the seed, each necessary to constitute the disease, but that the relative share which they appear to take varies in importance in different diseases. We as practical physicians have to obtain our knowledge as to the micro-organisms concerned in these diseases and their chemio-physiological action from those who devote themselves to these studies, whilst we have to observe the effects of the micro-organisms on the human body and how the body reacts to the germs of the disease. The infective diseases existed long before the discovery of the germs and we have accumulated a large store of knowledge of the clinical course, the treatment, and the issue of these diseases. This accumulated experience is of great value though apt to be overlooked by the keen interest excited by fresh discoveries. It is against this tendency that I wish to enter a protest and to point out that what is true is not necessarily new. We must never forget that the soil is of as great importance to study as is the seed in the proper understanding and treatment of diseases of microbic origin.

Health of St. Ives.-Mr. J. M. Nicholls, the medical officer of health of St. Ives, Cornwall, in his annual report for 1901 states that 197 births were registered during the year, corresponding to a rate of 29.41 per 1000 . deaths numbered 129, equal to a rate of $19 \cdot 26$ per 1000 .

\section{A Clinital andeture}

OY A CASE OF

\section{SUPPOSED INTESTINAL OBSTRUCTION, DUE TO A VASCULAR LESION,}

RAPIDLY FATAL FROM INOIPIENT GANGRENE OF THE STOMACH AND THE DUODENUM.

Delivered at St. Mary's Hospital on Feb. 25th, 1902.

\section{Br HERBERT W. PAGE, M.C. Cantab.,} SUBGEON TO THE HOSPTTAL.

GENTLEMEN, - I promised you a fortnight ago that I would tell you something about a remarkable case of intestinal obstruction which had been under my care recently. The case was one of singular interest and as far as my researches have carried me I believe it to have been unique. You will hear that there was no true intestinal obstruction at all, but nevertheless the case must be so described, for intestinal obstruction was a prominent feature of it and it was intestinal obstruction that I was called upon to relieve. Were I to ask you for the causes of intestinal obstruction you would doubtless be readily able to give me a list, as, for example, obstruction by band, by volvulus or kink, by growths which narrow the lumen of the bowel, by impaction of a foreign body, by accumulation of fæces, by any form of strangulation whether external and visible or internal and out of sight, and these various conditions you might speak of as true causes of obstruction in that they severally interfered with the lumen of the bowel and prevented the onward passage of its contents. And if I pressed you further you might remember other causes which rather may be regarded as those of false obstruction in that there is no mechanical interference with the lumen of the gut-the paralysis of bowel, for example, such as you may meet with from sundry nervous conditions, or the distension which may arise in the course of peritonitis when all peristalsis has been lost. Your list would be fairly complete, but I doubt whether you would think of vascular lesion alone as being a possible cause of intestinal obstruction. And yet there have been cases recorded from time to time where a vascular lesion-thrombosis of the superior mesenteric vein, for example-has given rise to symptoms of intestinal obstruction for which operation has been undertaken. I shall presently refer to a case of this sort which was brought before the Clinical Society of London by Dr. J. Rose Bradford and there have been other cases like it. ${ }^{2}$ We ought, therefore, to have this cause of intestinal obstruction in mind, although from its exceeding rarity we may hardly expect to meet with a case ourselves. Now let me tell you of my own case.

On Monday, Feb. 10th, I was sent for by my friend and former house surgeon, Dr. J. A. Wright, to see a patient with intestinal obstruction and to come prepared to operate. I went and this was the story which I was teld. The patient, a man aged 50 years, thin and spare, had enjoyed the best of health until three and a half years previously, when he had a very bad attack of typhoid fever which kept him in bed for some 10 weeks and which was complicated by thrombosis in the right leg. His health had never been the same since; he had lost his former strength and his capacity for continuous work. In the summer of 1899 he had thrombosis in the left leg following a bicycle accident and again in the spring of 1901 he was laid up for a month with thrombosis of the left femoral vein. I ask your special attention to these several attacks of thrombosis for they go far to explain his present illness. A week previously he had had rather a bad cold and had stayed indoors, but on the afternoon of Feb. 9th (Sunday) he was able to go out for a country walk. During the walk he was suddenly seized with pain above the umbilicus and this was soon followed by diarrhcea. As a matter of fact he had to relieve himself behind a hedge more than once, and

Notably since this lecture was given the case of "Intestinal Obstruction due to Embolism of a Branch of the Superior Mesenteric Obstruction due to Embolism of a Branch of the Superior Mesenteric Artery "brought before the Clinical Society of London by my friends Dr. W. J. Tyson and Mr. W. W. Linington of Folkestone, on April 1lth (see THE LANCET, April 19th, 1902, p. 1109). Coffee-ground vomit was
there also a prominent symptom. 